\title{
Early detection and quantification of cerebral venous thrombosis by Magnetic Resonance Black Blood Thrombus Imaging (MRBTI)
}

\author{
Qi Yang ${ }^{1 *}$, Zhaoyang Fan ${ }^{1}$, Xiaoming $\mathrm{Bi}^{2}$, Debiao $\mathrm{Li}^{1}$ \\ From 19th Annual SCMR Scientific Sessions \\ Los Angeles, CA, USA. 27-30 January 2016
}

\section{Background}

Early diagnosis of cerebral venous and sinus thrombosis (CVT) is currently a major clinical challenge. We proposed a selective MR black-blood thrombus imaging technique(MRBTI).

\section{Methods}

MRBTI was performed on 23 patients with proven CVT and 24 patients with negative CVT by conventional imaging techniques. Signal-to-noise ratio (SNR) was calculated for the detected thrombus and contrast-to-noise ratio (CNR) was measured between thrombus and lumen, and also between thrombus and brain tissue. The feasibility of using MRBTI for thrombus volume measurement was also explored.

\section{Results}

With effectively suppressed blood signal, MRBTI correctly identified 113 out of 116 segments with proven CVT with a sensitivity of $97.4 \%$. In 527 out 531 segments, CVT was ruled out correctly with a specificity of $99.3 \%$. Quantification of thrombus volume was successfully conducted in all patients with $\mathrm{CVT}$, and mean volume of thrombus was $10.5 \pm 6.9 \mathrm{cc}$.

\section{Conclusions}

The current findings support that MRBTI allows direct selective visualization of thrombus as opposed to indirect detection of venous flow perturbation and can be used as a promising first line diagnostic imaging tool.

\author{
Authors' details \\ ${ }^{1}$ Biomedical Imaging Research Institute, Cedars Sinai Medical Center, Los \\ Angeles, CA, USA. ${ }^{2}$ MR R\&D, Siemens Healthcare, Los Angeles, CA, USA.
}

Published: 27 January 2016

doi:10.1186/1532-429X-18-S1-P16

Cite this article as: Yang et al: Early detection and quantification of cerebral venous thrombosis by Magnetic Resonance Black Blood Thrombus Imaging (MRBTI). Journal of Cardiovascular Magnetic Resonance 2016 18(Suppl 1):P16. 


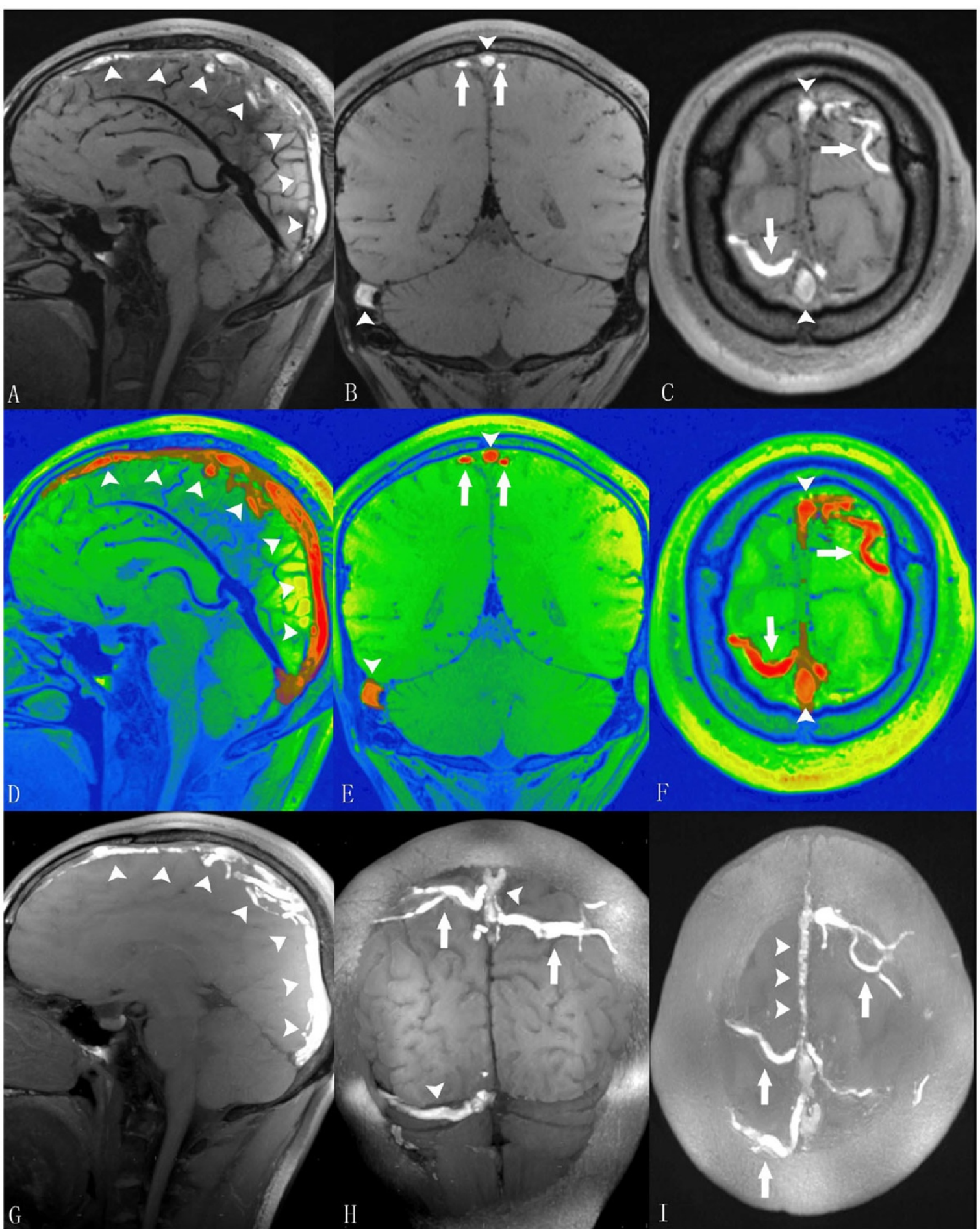

Figure 1 MRBTI of a 27-year-old male patient with sub-acute CVT. A-C: MRBTI demonstrated hyper-intense signal intensity in the superior sagittal sinus (arrowheads), the right transverse and sigmoid sinuses (arrowheads), and the cortical veins (arrows) suggesting intraluminal thrombus formation. D-F: All thrombi semi-automatically outlined by software based on their high signal contrast were rendered with red color and volume was $21.5 \mathrm{cc}$. G-I: sagittal, coronal and axial sections of maximum intensity projection (MIP) reformations of MRDTI better depicted the thrombosed segments with hyper-intense signals. 